\title{
Experimental study of high frequency stochastic resonance in Chua circuits
}

\author{
Iacyel Gomes ${ }^{1}$, Claudio R. Mirasso ${ }^{1}$, Raúl Toral ${ }^{1,2}$ \\ and O. Calvo ${ }^{1}$ \\ ${ }^{1}$ Departament de Física, Universitat de les Illes Balears, E-07122 Palma de \\ Mallorca, Spain \\ 2 Instituto Mediterráneo de Estudios Avanzados, IMEDEA (CSIC-UIB), E-07122 \\ Palma de Mallorca, Spain
}

\begin{abstract}
We study the stochastic resonance phenomenon occurring in electronic Chua circuits operating in the chaotic regime when the forcing signal is modulated at relatively high frequency, of the order of magnitude of the main Chua frequency in the absence of forcing $\left(f_{o}\right)$. In all the cases, a clear maximum in the signal-to-noise ratio for an intermediate noise level is observed. When modulating with a frequency smaller than $f_{o}$ we also observe a resonance at a harmonic of the external frequency which is closer to $f_{o}$ or to one of its harmonic. If the modulating frequency is larger than $f_{o}$ we only observe the resonance at the modulating frequency.
\end{abstract}

Key words: Stochastic Resonance, noise, Forced oscillators

PACS: 05.45.-a, 05.40.Ca, 05.45.Ac

Stochastic resonance is a nonlinear effect that accounts for the optimum response of a dynamical system to an external forcing at a precise value of the noise level. Since its original proposal as a mechanism to explain the observed periodicity of the Earth's ice ages, it has been found in a very large number of systems of physical, as well as biological, chemical, etc, interest[1,2,3]. A related effect is that of coherence resonance where the optimum response (in the sense of optimal periodicity) also occurs for a precise value of the noise level but in the absence of an external forcing[4]. Both effects have been observed in excitable as well as chaotic systems $[5,6]$, amongst others.

In this paper we experimentally study stochastic resonance on a Chua circuit operating in the chaotic regime. We consider the transitions from one scroll attractor to its mirror image induced by an external periodic forcing when the system operates under the effect of noise. At variance with similar studies, we 
chose a relatively large frequency, $f$, for the external modulation, of the order of magnitude of the main frequency, $f_{o}$, of the unperturbed Chua circuit. For all frequencies considered we observe a maximum in the signal-to-noise ratio at frequency $f$, a clear indication that stochastic resonance is taking place. Moreover, for forcing frequencies $f$ smaller than the unperturbed Chua frequency $f_{o}$ we observe stochastic resonance also at the harmonics of the modulating frequency $f$.
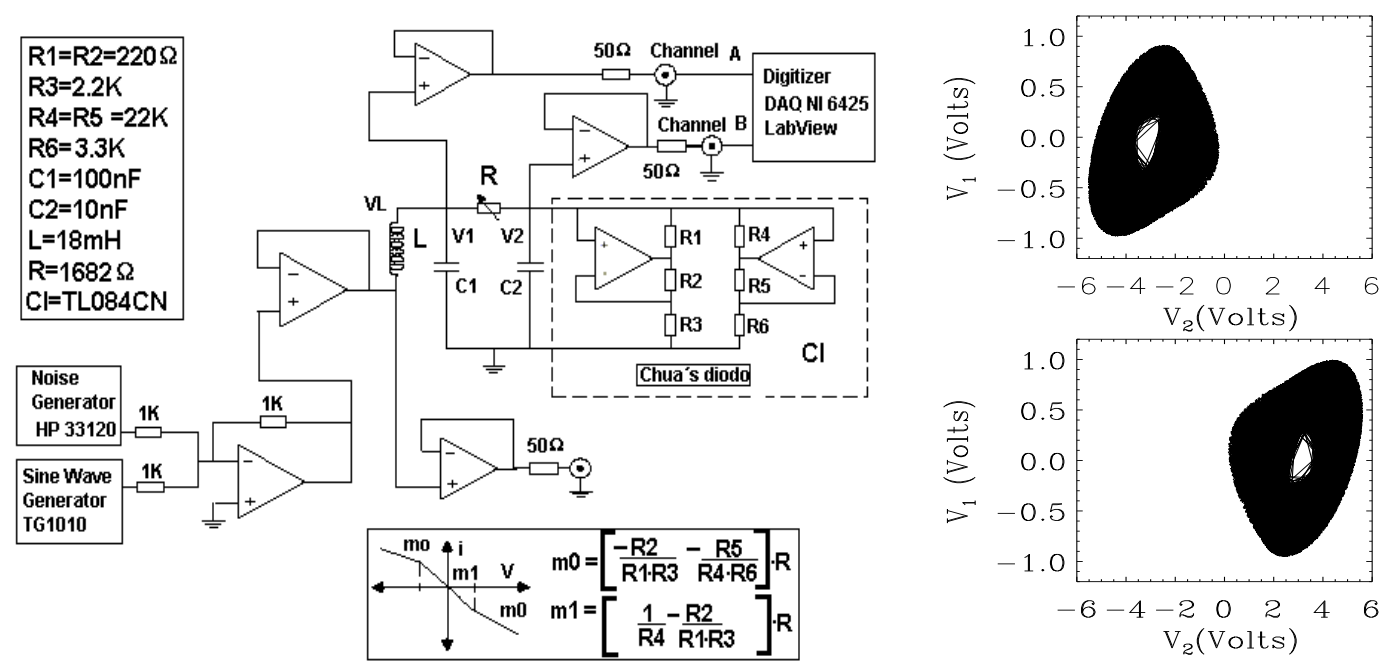

Fig. 1. Left panel: implemented Chua Circuit; right panel: single scroll chaotic attractor (upper panel) and mirror image (lower panel).

Our Chua circuit is schematically shown in fig. 1. A digital acquisition board from National Instruments NI-DAQ plugged into a computer was used to digitize the signal at a sampling rate of $10 \mathrm{KHz}$. A Labview program controlled the board in a continuous acquisition mode and an HP-33120 function generator was used to provide the noise signals of intensity $D$ ranging from 0 to $5 \mathrm{~V}[\mathrm{rms}]$. A signal generator is used to force the system with periodic forcing of the form $V(t)=V_{o} \sin (2 \pi f t)$, being $V_{o}$ the amplitude of the force and $f$ its frequency.

This Chua circuit can be described in terms of three non-linear first order differential equations[7], including those for the time evolution of the output voltages $V_{1}(t)$ and $V_{2}(t)$ (see fig. 1). These equations predict that, for some range of parameters, and in the absence of external forcing, the system can have several unstable fixed points such that the pair $\left(V_{1}(t), V_{2}(t)\right)$ oscillates chaotically in time around the corresponding dynamical attractors. For the set of parameters we have chosen in our experiments (see inset of fig. 1), two unstable fixed points lead to an isolated single scroll attractor and its mirror image (see fig. 1). The main frequency of the oscillations of the unperturbed 
circuit about each attractor is $f_{o} \sim 2.7 \mathrm{KHz}$. This is clearly indicated as a maximum in the power spectrum of the system, see fig. 2. Besides the characteristic frequency $f_{o}$, one can observe the appearance of several peaks at other relatively high frequencies, not far from $f_{o}$,
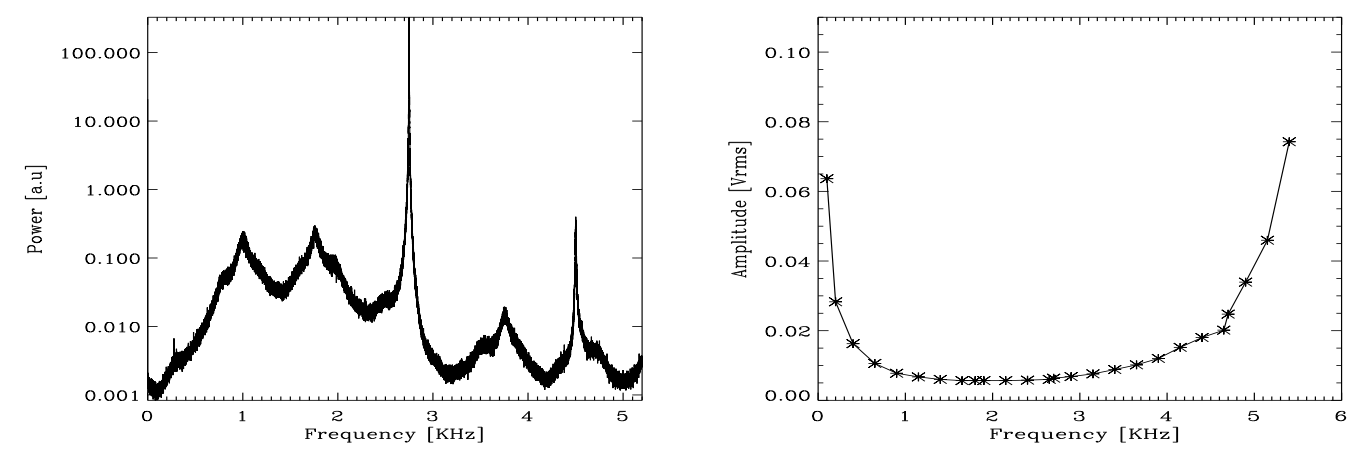

Fig. 2. Left panel: Power spectrum of the system in the absence of the external forcing. Right panel: Threshold value for the amplitude of the external forcing $V_{o}$ for the appearance of jumps between the single scroll attractors as a function of the forcing frequency $f$.

Note that for the chosen set of parameters, and without any external forcing, there is no possibility to jump from one attractor to the other, and which attractor is chosen depends exclusively on initial conditions. The presence of an external periodic forcing, however, can induce jumps between the attractors. For such an external forcing (but still in the absence of noise) a minimum value $V_{\text {min }}$ of its amplitude $V_{o}$ is required to induce those jumps. This minimum value is a degree of the sensitivity of the system to the external forcing and it is also plotted in fig. 2 as a function of the frequency $f$ of the forcing. It can be seen that close to the main unperturbed Chua frequency $f_{o}$ the amplitude is minimum and that it increases for smaller and larger frequencies.

With this information at hand, for each forcing frequency $f$ we now set the amplitude of the external forcing close, but below, the minimum value $V_{\text {min }}$ defined before. Under these conditions, the inclusion of noise of very small amplitude is able to induce jumps between the attractors. It is in the periodicity of these jumps where one can observe a resonance effect with respect to the noise intensity. We have focused our study in the cases where the external frequency coincides with some of the major peaks in the power spectrum of the unperturbed Chua system as shown in fig. 2. Namely, we have chosen $f=0.9 \mathrm{KHz}, f=1.8 \mathrm{KHz}, f=2.7 \mathrm{KHz}$ and $f=4.5 \mathrm{KHz}$. Note that the possibility of having stochastic resonance in a chaotic circuit with two attractors under the presence of noise has been shown theoretically and numerically by Anishchenko and coworkers[8,9]. This case is the the so-called deterministic stochastic resonance which appears in deterministic chaotic systems close to the onset of intermittency, i.e., a situation different to the one considered here. 

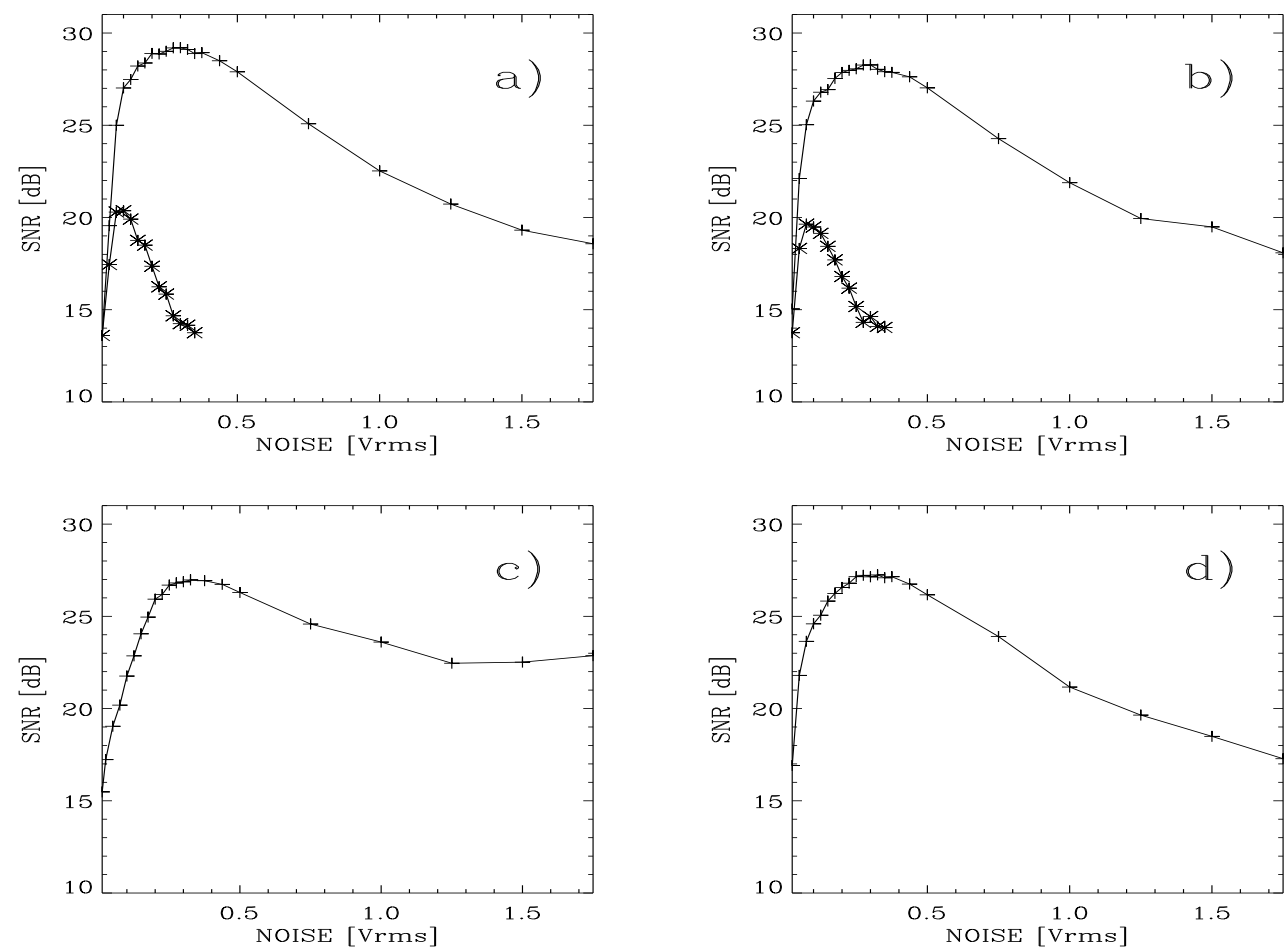

Fig. 3. Signal-to-noise ratio (SNR) vs. noise level for different forcing amplitudes: (a) $f=0.9 \mathrm{KHz}, V_{o}=7 \mathrm{mV}_{\mathrm{rms}}$; (b) $f=1.8 \mathrm{KHz}, V_{o}=5 \mathrm{mV}_{\mathrm{rms}}$; (c) $f=2.7 \mathrm{KHz}, V_{o}=6.4$ $\mathrm{mV}_{\mathrm{rms}}$ and d) $f=4.7 \mathrm{KHz}, V_{o}=25 \mathrm{mV}_{\mathrm{rms}}$. In panels (a) and (b) crosses stand for the response at the forcing frequency and stars for the response at its third harmonic. Solid lines are included to aid the eye.

Under modulation and noise, the power spectrum varies the relative height of their peaks. We characterize the response by the usual signal to noise ratio indicator. In fig. 3 we plot the results we obtain when modulating with different frequencies. In all the panels it can be clearly seen that even at these high frequencies a maximum of the signal-to-noise ratio is observed for an intermediate noise level, the most important signature of stochastic resonance. Moreover, the maxima occur for approximately the same noise level independently of the forcing frequency. Interestingly, when looking at the response at frequencies $f$ corresponding to other peaks in the power spectrum, we observe that for frequencies smaller than the main Chua frequency $f_{o}$ the system also exhibits stochastic resonance at harmonics of this forcing frequency $f$. It appears that stochastic resonance appears only for those harmonics whose frequency is closer to $f_{o}$ or to a harmonic of $f_{o}$. For example, in fig.4 panel a) another stochastic resonance effect is observed in the third harmonic of the forcing frequency (in this case $3 f=2.7 \mathrm{KHz} \approx f_{o}$ ) while in panel b) an stochastic resonance effect occurs at $3 f=5.4 \mathrm{KHz}$, which corresponds to $2 f_{o}$. On the contrary, when the external frequency is larger than $f_{o}$, we only observe stochastic resonance at the forcing frequency. 
In conclusion, we have experimentally shown that stochastic resonance in an electronic Chua circuit occurs even when the system is modulated at relatively high frequencies, as compared to the usual stochastic resonance in which the forcing frequency is small compared with any internal frequency. When forcing with a frequency smaller than the main unperturbed Chua frequency we have also shown that the resonance occurs not only at the forcing frequency $f$ but also at harmonics of $f$ which are close to the main unpertubed Chua frequency $f_{o}$ or to a harmonic of it.

The work is supported by MCyT (Spain) and FEDER, projects BFM20010341-C02-01, BMF2000-1108 and BFM2002-04369. I.G. acknowledges support form the Agencia Española de Cooperación Internacional.

\section{References}

[1] R. Benzi, A. Sutera and A. Vulpiani, J. Phys. A14, 453 (1981).

[2] C. Nicolis and G. Nicolis, Tellus 33, 225 (1981).

[3] L. Gammaitoni, P. Hänggi, P. Jung and F. Marchesoni, Rev. Mod. Phys. 70, 223 (1998).

[4] A.S. Pikovsky and J. Kurths, Phys. Rev. Lett. 78, 775 (1997).

[5] C. Palenzuela, R. Toral, C. Mirasso, O. Calvo and J. Gunton, Europhys. Lett. 56, 347 (2001).

[6] O. Calvo, C. Mirasso and R. Toral, ELectron. Lett. 37, 1062 (2001).

[7] Chua's Circuit: A Paradigm for Chaos, R.N. Madan, ed. World Scientific Publishing (1993).

[8] V.S. Anishchenko, M.A. Safanova and L.O. Chua, Int.J. Bif. Chaos 2, 397 (1992).

[9] V.S. Anishchenko, A.B. Neiman, F. Moss and L. Schimansky-Geier, PhysicsUspekhi 42, 7 (1999). 